\title{
A novel bicyclic 2,4-diaminopyrimidine inhibitor of Streptococcus suis dihydrofolate reductase
}

\author{
Warangkhana Songsungthong ${ }^{\text {Corresp., } 1}$, Sunisa Prasopporn ${ }^{1,2}$, Louise Bohan ${ }^{1,3}$, Potjanee Srimanote ${ }^{4}$, Ubolsree \\ Leartsakulpanich $^{1}$, Suganya Yongkiettrakul ${ }^{1}$ \\ ${ }^{1}$ Biosensing and Bioprospecting Research Group, National Center for Genetic Engineering and Biotechnology (BIOTEC), National Science and Technology \\ Development Agency (NSTDA), Pathum Thani, Thailand \\ 2 Department of Pharmacology, Faculty of Medicine, Siriraj Hospital, Mahidol University, Bangkok, Thailand \\ 3 School of Pharmacy and Life Sciences, The Robert Gordon University, Aberdeen, United Kingdom \\ 4 Faculty of Allied Health Sciences, Thammasat University, Klong Luang, Pathum Thani, Thailand \\ Corresponding Author: Warangkhana Songsungthong \\ Email address: warangkhana.son@biotec.or.th
}

Streptococcus suis is a Gram-positive bacterial pathogen of pigs and an emerging zoonotic pathogen. It has become increasingly resistant to multiple classes of antibiotics. New drug candidates and knowledge of their targets are needed to combat antibiotic-resistant $S$. suis. In this study, the open-source Pathogen Box compound library was screened. Thirty hits that effectively inhibited $S$. suis growth at $10 \mu \mathrm{M}$ were identified. Among the most potent hits, MMV675968 (a diaminoquinazoline analog) was shown to target $S$. suis dihydrofolate reductase (SSDHFR) via 1) growth inhibition of an E. coli surrogate whose growth is dependent on exogenously expressed SSDHFR and 2) inhibition of in vitro SsDHFR activity. Thymidine supplement is able to reverse growth inhibition by MMV675968 in both E. coli surrogate and S. suis, indicating that a thymidine-related pathway is a major target of MMV675968. Comparison of MMV675968 with seven DHFR inhibitors representing different core structures revealed that bicyclic 2,4diaminopyrimidines with long and flexible side chains are highly effective in inhibiting SsDHFR and S. suis growth. MMV675968 and related compounds thus may serve as starting points for developing antibiotics against drug resistant $S$. suis. 


\section{A novel bicyclic 2,4-diaminopyrimidine inhibitor of Streptococcus suis dihydrofolate 2 reductase \\ 3}

4 Warangkhana Songsungthong ${ }^{1}$, Sunisa Prasopporn ${ }^{1,3}$, Louise Bohan ${ }^{1,4}$, Potjanee Srimanote ${ }^{2}$, 5 Ubolsree Leartsakulpanich ${ }^{1}$, and Suganya Yongkiettrakul ${ }^{1}$

6

$7 \quad{ }^{1}$ Biomolecular Analysis and Application Laboratory, National Center for Genetic Engineering 8 and Biotechnology (BIOTEC), Pathum Thani, Thailand

$9 \quad{ }^{2}$ Faculty of Allied Health Sciences, Thammasat University, Pathum Thani, Thailand.

$10{ }^{3}$ Current address: Department of Pharmacology, Faculty of Medicine, Siriraj Hospital, Mahidol

11 University, Bangkok, Thailand

$12{ }^{4}$ Current address: School of Pharmacy and Life Sciences, Robert Gordon University, Aberdeen,

13 United Kingdom

14

15

16 Corresponding Author:

17 Warangkhana Songsungthong ${ }^{1}$

18113 Thailand Science Park, Klong Luang, Pathum Thani, 12120, Thailand

19 Email address: Warangkhana.son@biotec.or.th 


\section{Abstract}

Streptococcus suis is a Gram-positive bacterial pathogen of pigs and an emerging

22 zoonotic pathogen. It has become increasingly resistant to multiple classes of antibiotics. New

23 drug candidates and knowledge of their targets are needed to combat antibiotic-resistant $S$. suis.

24 In this study, the open-source Pathogen Box compound library was screened. Thirty hits that

25 effectively inhibited S. suis growth at $10 \mu \mathrm{M}$ were identified. Among the most potent hits,

26 MMV675968 (a diaminoquinazoline analog) was shown to target S. suis dihydrofolate reductase

27 (SsDHFR) via 1) growth inhibition of an E. coli surrogate whose growth is dependent on

28 exogenously expressed SsDHFR and 2) inhibition of in vitro SsDHFR activity. Thymidine

29 supplement is able to reverse growth inhibition by MMV675968 in both E. coli surrogate and $S$.

30 suis, indicating that a thymidine-related pathway is a major target of MMV675968. Comparison

31 of MMV675968 with seven DHFR inhibitors representing different core structures revealed that

32 bicyclic 2,4-diaminopyrimidines with long and flexible side chains are highly effective in

33 inhibiting SsDHFR and S. suis growth. MMV675968 and related compounds thus may serve as

34 starting points for developing antibiotics against drug resistant S. suis. 


\section{Introduction}

36 It is estimated that antibiotic resistance will lead to 10 million deaths per year and

37 economic loss of US $\$ 100$ trillion by the year 2050 (O’Neill, 2014). Alarmingly, bacteria

38 resistant to last resort antibiotics and to all commercially available antibiotics have already

39 emerged and spread, signaling the start of a global public health crisis (McGann et al., 2016;

40 Ruzauskas \& Vaskeviciute, 2016; McCarthy, 2017). New antibiotics, preferably with new

41 chemical scaffolds to bypass existing resistance mechanisms, are urgently needed. Pathogen

42 Box, an open-source library of 400 compounds with chemical structures distinct from currently

43 available antibiotics (www.pathogenbox.org), demonstrated activity against selected neglected

44 disease pathogens and low toxicity against human cells is a promising source of compounds for 45 antibiotic discovery.

46 Streptococcus suis is a Gram-positive bacterium that can cause severe symptoms such as

47 meningitis, septicemia, and arthritis in pigs and thus pose a high economic burden for the global

48 pig industry (Wertheim et al., 2009a). Zoonotic transmission to humans occurs from eating

49 uncooked contaminated pork or from occupation-related infection such as when farmers or

50 abattoir workers come into contact with infected pigs or pig carcasses (Wertheim et al., 2009a;

51 Ho et al., 2011; Goyette-Desjardins et al., 2014; Huong et al., 2014). S. suis isolates found in

52 pigs and in human cases in Vietnam and China are of the same serotype and multi locus

53 sequence typing (MLST), suggesting direct transmission (Hoa et al., 2011). In human infection,

54 S. suis causes meningitis, sepsis, arthritis, vestibular dysfunction, and permanent hearing loss

55 with fatality rate of approximately $13 \%$ (Huong et al., 2014). S. suis infection is a major cause of

56 bacterial meningitis in Southeast Asia, and was responsible for a Streptococcal Toxic Shock

57 Syndrome outbreak in China (Suankratay et al., 2004; Hui et al., 2005; Tang et al., 2006; Thi 
58 Hoang Mai et al., 2008; Wertheim et al., 2009c). The number of human S. suis cases has

59 increased recently, especially in areas with high density pig farming, making $S$. suis infection an

60 important emerging disease (Wertheim et al., 2009a; Gajdács et al., 2020).

61 Owing to widespread use of antibiotics for prophylaxis and as growth promoter in the pig

62 farming industry, antibiotic resistance in S. suis is rising (Wertheim et al., 2009a; Varela et al.,

63 2013; Hernandez-Garcia et al., 2017; Yongkiettrakul et al., 2019). S. suis isolates are recently

64 reported to be resistant to multiple classes of antibiotics such as $\beta$-lactams (e.g. ampicillin,

65 penicillin), macrolides (e.g. erythromycin and clarithromycin), lincosamide (e.g. clindamycin),

66 tetracycline, fluoroquinolone (e.g. moxifloxacin), aminocyclitol (e.g. spectinomycin),

67 aminoglycoside (e.g. gentamycin), and trimethoprim-sulfonamide (Yongkiettrakul et al., 2019;

68 Tan et al., 2020; Riley et al., 2020; Gajdács et al., 2020). Multidrug resistance in S. suis thus

69 prompts the need for new drugs. In this study, we screened the Pathogen Box library against two

70 strains of S. suis, namely P1/7 and HE06 (Jacobs, van den Berg \& Loeffen, 1996; Maneerat et

71 al., 2013) and identified 30 compounds with high growth inhibitory activity at $10 \mu \mathrm{M}$. The

72 potential target of MMV675968, a diaminoquinazoline derivative, was identified as

73 dihydrofolate reductase, which is a target of multiple classes of compounds including

74 antibacterial, antimalarial, and anticancer compounds (Gao et al., 2019). 


\section{Materials and Methods}

77 The experiments using S. suis was approved by BIOTEC Institutional Review Board on

78 biosafety and biosecurity with approval number BT-IBC-59-028.

\section{Bacteria strains and growth conditions}

80 S. suis strains $\mathrm{P} 1 / 7$ and HE06 are serotype 2 isolates obtained from a pig with meningitis and an

81 infected human, respectively (Jacobs, van den Berg \& Loeffen, 1996; Holden et al., 2009;

82 Maneerat et al., 2013). Serotype 2 strains were chosen because of their association with

83 pathogenicity (Lun et al., 2007; Wertheim et al., 2009b; Maneerat et al., 2013). S. suis was

84 grown on brain heart infusion (BHI) plates (Beckton Dickinson, Franklin Lakes, NJ, USA) at 37

$85{ }^{\circ} \mathrm{C}$ with $5 \% \mathrm{CO}_{2}$. Escherichia coli PA414 lacking folA and thyA genes, encoding dihydrofolate

86 reductase (DHFR) and thymidylate synthase (TS), respectively (Ahrweiler \& Frieden, 1988) was

87 used as a surrogate for expressing S. suis DHFR. The deletion of thyA is required for survival of

88 DHFR deficient mutant (Ahrweiler \& Frieden, 1988). E. coli PA414 transformed with various

89 expression plasmids was grown in Luria Bertani (LB) broth supplemented as necessary with 50

$90 \mu \mathrm{g} / \mathrm{mL}$ kanamycin, $100 \mu \mathrm{g} / \mathrm{mL}$ ampicillin, $10 \mu \mathrm{g} / \mathrm{mL}$ chloramphenicol, $0.2 \%$ (w/v) arabinose,

91 and $/$ or $50 \mu \mathrm{g} / \mathrm{mL}$ thymidine.

\section{Bacterial growth inhibition assay}

93 The Clinical Laboratory Standard Institute (CLSI) broth microdilution method was used to test

94 antibacterial activity of Pathogen Box compounds and selected DHFR inhibitors (The Clinical

95 and Laboratory Standards Institute, 2018). Briefly, 5 x $10^{4}$ colony forming units (CFU) of S. suis

96 or E. coli PA414 were incubated with compounds at $10 \mu \mathrm{M}$ (or other concentrations as

97 indicated) in cation-adjusted Mueller Hinton broth (Beckton Dickinson, Franklin Lakes, NJ, 
98 USA) in 96-well plates at $37{ }^{\circ} \mathrm{C}$ with or without $5 \% \mathrm{CO}_{2}$, respectively. Growth was monitored

99 by measuring optical density at $600 \mathrm{~nm}\left(\mathrm{OD}_{600}\right)$ using a SpectraMax M5 spectrophotometer

100 (Molecular Devices, San Jose, CA, USA). Percent growth of each compound-treated bacteria

101 strain was calculated using the following formula:

102 (OD of compound treated bacteria $\times 100) /($ OD of DMSO treated bacteria)

\section{Construction of expression plasmids}

104 The dhfr gene encoding S. suis dihydrofolate reductase (SsDHFR) was amplified from clarified

105 lysate of S. suis P1/7 and S. suis HE06 using high-fidelity Phusion DNA polymerase (New

106 England Biolabs, Ipswich, MA, USA) and primers

1075 'ctggtgecgegeggeagccatATGACTAAAAAGATTGTTGC3' and

1085 'tcgggctttgttagcagccggatcCTAACCATCTCTTCTTTCATAG3' (lower case denotes sequence

109 homologous to pET15b while upper case denotes sequence homologous to the $d h f r$ gene). The

110 PCR products were cloned into NdeI and BamHI digested pET15b plasmid using a Gibson

111 Assembly kit (New England Biolabs, Ipswich, MA, USA), according to manufacturer's protocol.

112 The resulting plasmids were subjected to Sanger sequencing ( $1^{\text {st }}$ BASE, Singapore). Nucleotide

113 sequences of the dhfr gene from S. suis P1/7 and S. suis HE06 were deposited in NCBI Genbank

114 with accession number MH388486 and MH388487, respectively.

\section{Complementation assay of $E$. coli surrogate}

116 E. coli PA414 lacking functional DHFR and TS enzymes (Ahrweiler \& Frieden, 1988) was used

117 as a surrogate host to study the function of exogenously expressed SsDHFR. E. coli PA414 was

118 co-transformed with 1) pBAD33 or pBAD33-EcTS and 2) pET15b or pET15b-SsDHFR. To test

119 whether SsDHFR can complement for the loss of E. coli DHFR in the E. coli surrogate, 
120 overnight cultures of E. coli PA414 carrying various combination of plasmids were pelleted,

121 washed, cell density adjusted to $\mathrm{OD}_{600}$ of 4 , serially-diluted, and spotted onto LB plates

122 supplemented with appropriate antibiotics and either $50 \mu \mathrm{g} / \mathrm{mL}$ thymidine or $0.2 \%(\mathrm{w} / \mathrm{v})$

123 arabinose. Plates were incubated at $37^{\circ} \mathrm{C}$ overnight. Growth on agar plate was observed.

\section{Overexpression of SsDHFR in E. coli BL21(DE3)}

125 Cultures of E. coli BL21(DE3) carrying pET15b or pET15b-SsDHFR were grown with shaking 126 at $37^{\circ} \mathrm{C}$ in $\mathrm{LB}$ supplemented with $100 \mu \mathrm{g} / \mathrm{mL}$ ampicillin. When $\mathrm{OD}_{600}$ reached 1 , IPTG was

127 added to $40 \mu \mathrm{M}$, temperature shifted to $16^{\circ} \mathrm{C}$, and incubated with shaking overnight. Bacterial

128 pellets were collected by centrifugation, resuspended in lysis buffer $(20 \mathrm{mM}$ potassium

129 phosphate buffer, $0.1 \mathrm{mM}$ EDTA, $10 \mathrm{mM}$ DTT, $50 \mathrm{mM} \mathrm{KCl}, 20 \%$ glycerol) and sonicated.

130 Protein concentration of clarified bacteria lysate was determined by Bradford assay (Biorad,

131 Hercules, CA, USA) according to the manufacturer's protocol.

\section{In vitro DHFR activity assay}

133 DHFR uses NADPH as a cofactor for the conversion of dihydrofolate (DHF) to tetrahydrofolate

134 (THF). Since the amount of NADPH consumed is directly proportional to the amount of THF

135 product, monitoring NADPH consumed using absorbance at $340 \mathrm{~nm}\left(\mathrm{~A}_{340} ; \varepsilon_{340}\right.$ of $12,300 \mathrm{M}^{-1}$

$136 \mathrm{~cm}^{-1}$ ) is indicative of DHFR enzymatic activity. The DHFR activity assay was performed as

137 previously described (Tirakarn et al., 2012; Songsungthong et al., 2019). Briefly, using kinetics

138 mode of a UV-Vis spectrophotometer (Agilent Technologies, Santa Clara, CA, USA), A 340 value

139 of the reaction mixture (50 mM TES, $\mathrm{pH}$ 7.0, $75 \mathrm{mM} \beta$-mercaptoethanol, $1 \mathrm{mM}$ EDTA, 1

$140 \mathrm{mg} / \mathrm{mL}$ BSA, $0.1 \mathrm{mM}$ NADPH, $0.1 \mathrm{mM}$ dihydrofolate $)$ was set to blank $\left(\mathrm{A}_{340}=0\right)$. Inhibitors

141 were added to the reaction mixture as necessary. $1 \mu \mathrm{g}$ of total protein from lysate of E. coli 
142 BL21(DE3) containing pET15b or overexpressing SsDHFR was added to reaction buffer to

143 initiate the enzymatic reaction. The amount of SsDHFR containing lysate added was

144 predetermined to give linear reaction kinetics. NADPH reduction was monitored by measuring

$145 \mathrm{~A}_{340}$ reduction for 100 seconds. $\mathrm{A}_{340}$ reduction per minute values were recorded.

146 Percent SsDHFR activity was calculated using the following formula:

$147\left(\mathrm{~A}_{340}\right.$ reduction per minute of compound-treated lysate $\left.\times 100\right) /\left(\mathrm{A}_{340}\right.$ reduction per minute of

148 DMSO-treated lysate of BL21 expressing SsDHFR)

\section{Statistical analysis}

150 Data are shown as mean \pm standard error of the mean (SEM). At least three independent

151 experiments were performed. Statistical analysis using one-way ANOVA with Tukey's post test

152 was performed. Statistical significance is noted on the graph by asterisks. * denotes $\mathrm{P}<0.05$.**

153 denotes $\mathrm{P}<0.01$. ** denotes $\mathrm{P}<0.001$. *** denotes $\mathrm{P}<0.0001$. ns denotes not statistically

154 significant. 


\section{Results}

\section{Identifying compounds with $S$. suis growth inhibitory activity from Pathogen Box}

158 We screened the Pathogen Box library for compounds active against $S$. suis P1/7 and $S$.

159 suis HE06 isolated from an infected pig and human, respectively (Jacobs, van den Berg \&

160 Loeffen, 1996; Holden et al., 2009; Maneerat et al., 2013). Thirty compounds with high

161 inhibitory activity (with average percent inhibition of $90-100 \%$ ) at $10 \mu \mathrm{M}$ were identified

162 (Table S1). Seven of the hits including rifampicin, levofloxacin, and linezolid are reference

163 compounds known to be broadly effective against Gram-positive bacteria (highlighted in blue in

164 Table S1). Twenty three other hits are compounds with structures distinct from commercially

165 available antibiotics (highlighted in green in Table S1). The mechanisms of action of most non-

166 reference hits are unknown. MMV675968, one of the hits, has been shown to inhibit the growth

167 of protozoan parasites and Gram-negative bacteria (Lau et al., 2001; Nelson \& Rosowsky, 2001;

168 Popov et al., 2006; Songsungthong et al., 2019) via inhibition of dihydrofolate reductase

169 (DHFR), an enzyme in the thymidylate cycle.

170 MMV675968 is an inhibitor of $S$. suis DHFR

171 MMV675968 inhibits DHFR of protozoan parasites and of Gram-negative bacteria (Lau et

172 al., 2001; Nelson \& Rosowsky, 2001; Popov et al., 2006; Songsungthong et al., 2019). We

173 therefore hypothesized that MMV675968 inhibited S. suis growth through inhibition of S. suis

174 DHFR (SsDHFR). An E. coli surrogate assay and an in vitro DHFR activity assay were used to

175 test this hypothesis. For the E. coli surrogate assay, E. coli PA414 deficient in E. coli DHFR

176 (EcDHFR) and E. coli thymidylate synthase ( $E c$ TS) of the thymidylate cycle making the strain a

177 thymidine auxotroph (Ahrweiler \& Frieden, 1988), was used as a host for exogenously 
178 expressing SsDHFR. E. coli PA414 transformant controls carrying two empty vectors (pET15b

179 and pBAD33), or expressing EcTS alone did not grow in the absence of thymidine supplement as

180 expected (Fig. 1A). E. coli PA414 expressing EcTS together with SsDHFR was able to grow

181 without thymidine supplement (Fig.1A), indicating that $S s$ DHFR was functional and

182 complemented for the loss of EcDHFR. Growth of E. coli PA414 in the absence of thymidine

183 was therefore dependent on the function of exogenously expressed SsDHFR.

184 Trimethoprim (TMP), a bacterial DHFR inhibitor (Baker et al., 1981), inhibited the growth

185 of E. coli PA414 expressing SsDHFR to $19 \%$ of untreated (Fig. 1B), confirming that

186 trimethoprim inhibits SsDHFR. MMV675968 was more effective than TMP as seen by its ability

187 to inhibit the growth of E. coli PA414 expressing SsDHFR to 7\% (Fig. 1B). E. coli PA414

188 growth inhibition by MMV675968 was reversed in the presence of thymidine supplement (Fig.

189 1C), suggesting that the thymidine-related pathway is the principal target of MMV675968 in $E$.

190 coli surrogate consistent with DHFR being a target.

191 Next, we tested whether MMV675968 could inhibit SsDHFR activity directly by an in

192 vitro DHFR activity assay. DHFR activity present in lysate of E. coli BL21(DE3) carrying an

193 empty pET15b plasmid accounted for only $2 \%$ of DHFR activity of lysate of $E$. coli BL21(DE3)

194 overexpressing SsDHFR (Fig. 1D), indicating that most of the DHFR activity observed was from

195 overexpressed SsDHFR rather than endogenous EcDHFR. In the presence of $1 \mu \mathrm{M}$

196 MMV675968, DHFR activity decreased to $2 \%$ of that of untreated lysate (Fig. 1D), indicating

197 that MMV675968 inhibits SsDHFR directly. Results from both E. coli surrogate assay and

198 DHFR activity assay thus indicate that MMV675968 inhibits SsDHFR.

199 We also tested whether thymidine-related pathway is the main target of MMV675968 in $S$.

200 suis by testing whether growth inhibition was rescued by thymidine supplement. Growth of both 
201 S. suis P1/7 and S. suis HE06 were inhibited in the presence of $1 \mu$ M MMV675968 (Fig. 1E).

202 Growth inhibition of both S. suis strains was reversed with thymidine supplement (Fig. 1E),

203 indicating that the main target of MMV675968 within S. suis is a thymidine-related pathway.

204 Bicyclic 2,4-diaminopyrimidines with long and flexible side chain associate with higher

205 inhibitory activity against $S$ sDHFR and $\boldsymbol{S}$. suis

206 To investigate which chemical scaffolds are better at inhibiting SsDHFR and S. suis

207 growth, eight DHFR inhibitors of various chemical structures (Fig. 2A-C), which include

208 commercially available compounds (pemetrexed, trimethoprim, pyrimethamine, cycloguanil, and

209 methotrexate), compounds from Medicines for Malaria Venture (MMV)'s Malaria Box

210 (MMV667486 and MMV667487), and a compound from Pathogen Box (MMV675968), were

211 tested for SsDHFR inhibitory activity and S. suis growth inhibition. Pemetrexed, MMV667486,

212 and MMV667487 did not show significant SsDHFR or S. suis growth inhibitory activity (Fig.

213 2A, D, E), indicating that these structures do not inhibit SsDHFR and consequently are unable to

214 inhibit S. suis growth. Trimethoprim, pyrimethamine, and cycloguanil were moderately effective

215 at inhibiting SsDHFR and S. suis growth (defined as being effective at $10 \mu \mathrm{M}$ but not at $1 \mu \mathrm{M}$ )

216 (Fig. 2B, D-F). Methotrexate and MMV675968 inhibited SsDHFR more effectively that

217 trimethoprim, and completely inhibited S. suis growth, even at $1 \mu \mathrm{M}$ (Fig. 2C-F). Methotrexate

218 and MMV675968, both of which are bicyclic 2,4-diaminopyrimidines with long and flexible side

219 chains, are highly effective inhibitors of both SsDHFR and S. suis growth. Both $S$. suis P1/7 and

220 S. suis HE06 strains shared a similar sensitivity pattern towards various DHFR inhibitors (Fig.

221 2E, F) consistent with the identical DHFR sequences between the two strains (Fig. S1 A-B). 


\section{Discussion}

224 Thirty compounds with high S. suis growth inhibitory activity were identified from the

225 Pathogen Box library (Table S1). Among the thirty hits, seven are reference antibiotics known to

226 be effective against Gram-positive bacteria, including levofloxacin, rifampicin, and linezolid

227 (highlighted in blue in Table S1). The chemical structures of twenty three other hits are distinct

228 from commercially available antibiotics (highlighted in green in Table S1). Approximately $60 \%$

229 of these hits (14 out of 23) also have activity against Mycobacterium tuberculosis

230 (www.pathogenbox.org), suggesting that they have broad activity against Gram-positive

231 organisms and are attractive as starting points for developing antibiotics.

232 Among the 23 hits with novel structures, compounds MMV688508 and MMV687813 are

233 oxazolidinone analogs. These compounds share the same core structure with radezolid, sutezolid,

234 and linezolid, which are known to have broad spectrum activity against Gram-positive bacteria

235 (Bozdogan \& Appelbaum, 2004). Oxazolidinones inhibit bacterial protein synthesis by binding

236 to the 50S ribosomal subunit and preventing the formation of a functional ribosome (Bozdogan

237 \& Appelbaum, 2004). It is therefore likely that MMV688508 and MMV687813 target S. suis

238 protein synthesis in a similar fashion. Besides inhibiting the growth of S. suis, MMV688508 also

239 inhibits the growth of other Gram-positive pathogens such as Mycobacterium tuberculosis,

240 Mycobacterium abscessus, and Staphylococcus aureus (Bhandari et al., 2018; Jeong et al., 2018),

241 showing broad-spectrum activity of MMV688508 against various Gram-positive pathogens.

242 Interestingly, MMV687813 is not reported to have anti-Staphylococcus activity or anti-M.

243 abscessus activity (Bhandari et al., 2018; Jeong et al., 2018) despite having activity against $M$.

244 tuberculosis and S. suis (www.pathogenbox.org, Table S1), pointing to structural differences 
245 among ribosomal targets and/or differences in compound permeability in various Gram-positive 246 species.

247 DHFRs are present in various organisms including protozoan parasites, human, and 248 bacteria. Even though DHFRs of different organisms perform the same function, i.e. the 249 conversion of dihydrofolate (DHF) to tetrahydrofolate (THF) using NADPH as a cofactor, 250 structures of DHFRs from different organisms are different and can be clustered into at least nine 251 distinct clades, resulting in different druggable space and different structures of effective 252 inhibitors (Bhosle \& Chandra, 2016). DHFRs from various bacteria species do not necessarily 253 cluster to the same clade. Specifically, SsDHFR does not belong in the same clade as DHFRs of

254 Gram-negative bacteria previously shown to be inhibited by MMV675968 (Nelson \& Rosowsky, 255 2001; Songsungthong et al., 2019). Consequently, it cannot be assumed a priori that

256 MMV675968 will effectively inhibit DHFR from S. suis. Experiment testing inhibition of 257 SsDHFR by MMV675968 was therefore needed.

258 This study is the first to show that MMV675968 has growth inhibitory activity against a 259 Gram-positive bacterium, namely $S$. suis, by inhibiting SsDHFR (Table S1, Fig. 1B, 1D), raising 260 a possibility that MMV675968 may serve as a broad-spectrum antibiotic candidate. Growth 261 inhibition by MMV675968 is reversed by thymidine supplement (Fig. 1C, 1E), confirming that 262 the principal target of MMV675968 is a thymidine-related pathway.

263 Eight DHFR inhibitors of various chemical structures were tested for their efficacy in 264 inhibiting SsDHFR and $S$. suis growth, revealing structures associated with ineffective, 265 moderately effective, and highly effective inhibitors (Fig. 2). Pemetrexed (a human DHFR 266 inhibitor), MMV667486, and MMV667487 (Plasmodium DHFR inhibitors (Aroonsri et al., 267 2016)) were ineffective as inhibitors against SsDHFR and S. suis (Fig. 2A, D, E). Trimethoprim, 
268 pyrimethamine, and cycloguanil, which are known inhibitors of bacterial and parasite DHFRs

269 (Gleckman, Blagg \& Joubert; Rollo, 1955; Tonelli et al., 2017), were moderately effective at

270 inhibiting SsDHFR and S. suis growth (Fig. 2B, D-F). These moderately-effective inhibitors

271 contain either diaminopyrimidine (trimethoprim and pyrimethamine) or diaminodimethyltriazine

272 (cycloguanil) cores with a rigid or flexible side chain. Interestingly, even though MMV667486,

273 MMV667487, and cycloguanil share the same diaminodimethyltriazine core and a phenyl ring at

274 the same position, MMV667486 and MMV667487 were ineffective at inhibiting SsDHFR and $S$.

275 suis growth whereas cycloguanil was effective (Fig. 2A, B, D, E). The data suggest that the

276 SsDHFR active site can accommodate the shorter side chain of cycloguanil, whereas the

277 longer/bulkier side chains of MMV667486 and MMV667487 may interfere with compound

278 binding to the SsDHFR active site, rendering the compounds ineffective as inhibitors. A

279 correlation between SsDHFR inhibitory activity and $S$. suis growth inhibition of various

280 compounds was observed (Fig. 2D-F), implying that the two S. suis strains are permeable to all

281 DHFR inhibitors tested allowing access to the $S s$ DHFR target, leading to growth inhibition. It is

282 possible that other S. suis strains may have different compound permeability patterns.

283 Among the inhibitors tested, MMV675968 and methotrexate were highly effective

284 SsDHFR and S. suis inhibitors (Fig. 2C-F), both of which contain a bicyclic 2,4-

285 diaminopyrimidine core with a long and flexible side chain. Such structures can serve as starting

286 points for further rational drug design against SsDHFR and S. suis. Streptococcus pneumoniae

287 DHFR is approximately $53 \%$ identical to SsDHFR and share high sequence homology at the

288 folate and NADPH binding sites (Fig.S1C), raising a possibility that methotrexate and

289 MMV675968 may also be effective against S. pneumoniae or other Streptococci. Other bicyclic

290 2,4-diaminopyrimidines were able to inhibit Streptococcus mutans growth (Zhang et al., 2015), 
291 confirming this hypothesis. Since methotrexate is a known inhibitor of human DHFR, whereas

292 MMV675968 shows 59-83 fold selectivity for bacterial DHFRs over human DHFR (Nelson \&

293 Rosowsky, 2001; Songsungthong et al., 2019), and is not cytotoxic against human HL-60 and

294 HepG2 cell lines (www.pathogenbox.org), MMV675968 might be a better starting compound for

295 antibiotic discovery.

\section{Conclusions}

297 A screen of the Pathogen Box compound library leads to identification of 23 new

298 bioactive compounds against S. suis (Table S1). MMV688508 and MMV687813, oxazolidinone

299 analogs, are strong inhibitors of $S$. suis growth (Table S1). Since MMV688508 is shown to

300 inhibit the growth of multiple Gram-positive species (Bhandari et al., 2018; Jeong et al., 2018),

301 MMV688508 may be able to serve as an antibiotic candidate against Gram-positive pathogens.

302 MMV675968, a bicyclic 2,-4 diaminopyrimidine with a long and flexible side chain, was

303 identified as a potent inhibitor of SsDHFR and of S. suis growth. MMV675968 may be able to

304 serve as a broad-spectrum antibiotic candidate since it is shown to inhibit the growth of both

305 Gram-positive and Gram-negative bacteria (Nelson \& Rosowsky, 2001; Songsungthong et al., 306 2019).

307

\section{Acknowledgments}

309 Pathogen Box and specific compounds in Pathogen Box and Malaria Box were obtained

310 from Medicines for Malaria Venture (www.pathogenbox.org). Plasmid pL0035 (MRA-850),

311 contributed by Andrew P. Waters, was obtained through the NIH Biodefense and Emerging

312 Infections Research Resources Repository Resources, NIAID, NIH. We thank Wildan Firdaus 
313 and Buppa Arechanajan for technical assistance, Penchit Chitnumsub for helpful comments, and 314 Philip J. Shaw for critical proof-reading of the manuscript. 


\section{Figure legends}

316

\section{Fig. 1. MMV675968 inhibits Streptococcus suis dihydrofolate reductase (SsDHFR). A)}

318 SsDHFR functions in E. coli PA414. Overnight cultures of E. coli PA414 carrying empty

319 plasmids or expressing EcTS and SsDHFR were serially diluted and spotted onto LB plates

320 supplemented with either $50 \mu \mathrm{g} / \mathrm{mL}$ thymidine or $0.2 \%$ (w/v) arabinose. Overnight growth was

321 recorded. A representative picture from three independent experiment is shown. B) MMV675968

322 inhibits SsDHFR in E. coli surrogate. E. coli PA414 expressing EcTS and SsDHFR was

323 incubated with DMSO, trimethoprim (TMP), or MMV675968 (10 $\mu$ M). Growth of compound-

324 treated E. coli surrogate was calculated as percent of untreated (100\%). C) Growth inhibition of

325 E. coli surrogate expressing SsDHFR by MMV675968 can be rescued by thymidine supplement.

326 E. coli PA414 expressing EcTS and SsDHFR was incubated with or without $10 \mu \mathrm{M}$

327 MMV675968 and with or without $50 \mu \mathrm{g} / \mathrm{mL}$ thymidine. Growth was monitored and compared

328 with that of untreated control (100\%). D) MMV675968 inhibits SsDHFR activity. In vitro

$329 S s$ DHFR activity from clarified lysate of E. coli BL21(DE3) overexpressing SsDHFR was

330 compared with that of E. coli BL21(DE3) harboring empty plasmid or in the presence of $1 \mu \mathrm{M}$

331 MMV675968. E) S. suis growth inhibition by MMV675968 can be rescued by thymidine

332 supplement. S. suis P1/7 and S. suis HE06 was incubated with or without $1 \mu \mathrm{M}$ MMV675968

333 and with or without $10 \mu \mathrm{g} / \mathrm{mL}$ thymidine. Growth was monitored and compared with that of

334 untreated control (100\%). The graphs (B-E) show mean \pm standard error of the mean (SEM)

335 from three independent experiments. One-way ANOVA with Tukey's post test was performed to

336 determine statistical significance compared with untreated control. *, $\mathrm{P}<0.05$; **, $\mathrm{P}<0.01$;

$337 * * * *, \mathrm{P}<0.0001 ;$ ns, not statistically significant. 
339 Fig. 2. Efficacy of various dihydrofolate reductase (DHFR) inhibitors of Streptococcus suis

340 DHFR (SsDHFR) and $S$. suis growth. Structures of eight DHFR inhibitors tested in this study

341 and grouped according to their inhibitory activity: A) ineffective inhibitors, B) moderately

342 effective inhibitors, and C) highly effective inhibitors. D) SsDHFR activity in the presence of 1

$343 \mu \mathrm{M}$ DHFR inhibitors. E) Percent growth of S. suis P1/7 (white bar) and S. suis HE06 (black bar)

344 after 18 hour incubation with $10 \mu \mathrm{M}$ of DHFR inhibitors. F) Percent growth of S. suis P1/7

345 (white bar) and S. suis HE06 (black bar) after 18 hour incubation with $1 \mu \mathrm{M}$ inhibitors. PMX,

346 pemetrexed; TMP, trimethoprim; PYR, pyrimethamine; MTX, methotrexate. The graphs show

347 mean \pm standard error of the mean (SEM) from at least three independent experiments. One-way

348 ANOVA with Tukey's post test was performed to determine statistical significance compared

349 with untreated control. *, $\mathrm{P}<0.05$; $^{* *}, \mathrm{P}<0.01 ; * * *, \mathrm{P}<0.001 ; * * * *, \mathrm{P}<0.0001$; ns, not

350 statistically significant. For Fig. 2E-F, black asterisks show statistical significance between

351 untreated and compound-treated S. suis $\mathrm{P} 1 / 7$ whereas red asterisks show statistical significance

352 between untreated and compound-treated S. suis HE06.

353 
354 Fig. S1. Alignment of $d h f r$ gene from S. suis P1/7 and HE06. A) Nucleotide sequence of

355 Ssdhfr gene (Genbank accession number MH388486 and MH388487) and B) amino acid

356 sequence of SsDHFR from S. suis P1/7 and S. suis HE06, and C) amino acid sequence of

357 SsDHFR and S. pneumoniae DHFR (SpDHFR) were aligned using Clustal Omega (Sievers \&

358 Higgins, 2014). Amino acids labeled in red are predicted to be folate binding site whereas amino

359 acids labeled in blue are predicted to be NADPH binding site by NCBI conserved domain

360 database (Marchler-Bauer et al., 2015).

361 


\section{References}

363 Ahrweiler PM, Frieden C. 1988. Construction of a fol mutant strain of Escherichia coli for use in

364 dihydrofolate reductase mutagenesis experiments. Journal of bacteriology 170:3301-4.

365 Aroonsri A, Akinola O, Posayapisit N, Songsungthong W, Uthaipibull C, Kamchonwongpaisan

366 S, Gbotosho GO, Yuthavong Y, Shaw PJ. 2016. Identifying antimalarial compounds

367 targeting dihydrofolate reductase-thymidylate synthase (DHFR-TS) by chemogenomic

368 profiling. International Journal for Parasitology 46:527-535. DOI:

$369 \quad 10.1016 /$ j.ijpara.2016.04.002.

370 Baker DJ, Beddell CR, Champness JN, Goodford PJ, Norrington FEA, Smith DR, Stammers

371 DK. 1981. The binding of trimethoprim to dihydrofolate reductase.

372 Bhandari V, Chakraborty S, Brahma U, Sharma P. 2018. Identification of Anti-staphylococcal

373 and Anti-biofilm Compounds by Repurposing the Medicines for Malaria Venture Pathogen

374 Box. Frontiers in cellular and infection microbiology 8:365. DOI:

$375 \quad 10.3389 /$ fcimb. 2018.00365.

376 Bhosle A, Chandra N. 2016. Structural analysis of dihydrofolate reductases enables

377 rationalization of antifolate binding affinities and suggests repurposing possibilities. FEBS

378 Journal 283:1139-1167. DOI: 10.1111/febs.13662.

379 Bozdogan B, Appelbaum PC. 2004. Oxazolidinones: Activity, mode of action, and mechanism

380 of resistance. International Journal of Antimicrobial Agents 23:113-119. DOI:

$381 \quad$ 10.1016/j.jijantimicag.2003.11.003.

382 Gajdács M, Németh A, Knausz M, Barrak I, Stájer A, Mestyán G, Melegh S, Nyul A, Tóth Á,

383 Ágoston Z, Urbán E. 2020. Streptococcus suis: An Underestimated Emerging Pathogen in 
384 Hungary? Microorganisms 8:1292. DOI: 10.3390/microorganisms8091292.

385 Gao T, Zhang C, Shi X, Guo R, Zhang K, Gu J, Li L, Li S, Zheng Q, Cui M, Cui M, Gao X, Liu

386 Y, Wang L. 2019. Targeting dihydrofolate reductase: Design, synthesis and biological

387 evaluation of novel 6-substituted pyrrolo[2,3-d]pyrimidines as nonclassical antifolates and

388 as potential antitumor agents. European Journal of Medicinal Chemistry 178:329-340.

389 DOI: $10.1016 /$ j.ejmech.2019.06.013.

390 Gleckman R, Blagg N, Joubert DW. Trimethoprim: mechanisms of action, antimicrobial activity,

391 bacterial resistance, pharmacokinetics, adverse reactions, and therapeutic indications.

392 Pharmacotherapy 1:14-20.

393 Goyette-Desjardins G, Auger JP, Xu J, Segura M, Gottschalk M. 2014. Streptococcus suis, an

394 important pig pathogen and emerging zoonotic agent-an update on the worldwide

395 distribution based on serotyping and sequence typing. Emerging Microbes and Infections 3.

396 DOI: $10.1038 / \mathrm{emi} .2014 .45$.

397 Hernandez-Garcia J, Wang J, Restif O, Holmes MA, Mather AE, Weinert LA, Wileman TM,

398 Thomson JR, Langford PR, Wren BW, Rycroft A, Maskell DJ, Tucker AW. 2017. Patterns

399 of antimicrobial resistance in Streptococcus suis isolates from pigs with or without

400 streptococcal disease in England between 2009 and 2014. Veterinary Microbiology

$401 \quad 207: 117-124$. DOI: 10.1016/j.vetmic.2017.06.002.

402 Ho DTN, Le TPT, Wolbers M, Cao QT, Nguyen VMH, Tran VTN, Le TPT, Nguyen HP, Tran 403 THC, Dinh XS, To SD, Hoang TTH, Hoang T, Campbell J, Nguyen VVC, Nguyen TC, 404 Nguyen VD, Ngo TH, Spratt BG, Tran TH, Farrar J, Schultsz C. 2011. Risk Factors of 405 Streptococcus suis Infection in Vietnam. A Case-Control Study. PLoS ONE 6:e17604. DOI: 
$406 \quad$ 10.1371/journal.pone.0017604.

407 Hoa NT, Chieu TTB, Nga TTT, Dung N Van, Campbell J, Anh PH, Huu Tho H, Van Vinh Chau 408 N, Bryant JE, Hien TT, Farrar J, Schultsz C. 2011. Slaughterhouse Pigs Are a Major 409 Reservoir of Streptococcus suis Serotype 2 Capable of Causing Human Infection in $410 \quad$ Southern Vietnam. PLoS ONE 6:e17943. DOI: 10.1371/journal.pone.0017943.

411 Holden MTG, Hauser H, Sanders M, Ngo TH, Cherevach I, Cronin A, Goodhead I, Mungall K, 412 Quail MA, Price C, Rabbinowitsch E, Sharp S, Croucher NJ, Chieu TB, Thi Hoang Mai N, 413 Diep TS, Chinh NT, Kehoe M, Leigh JA, Ward PN, Dowson CG, Whatmore AM, Chanter 414 N, Iversen P, Gottschalk M, Slater JD, Smith HE, Spratt BG, Xu J, Ye C, Bentley S, Barrell 415 BG, Schultsz C, Maskell DJ, Parkhill J. 2009. Rapid Evolution of Virulence and Drug 416 Resistance in the Emerging Zoonotic Pathogen Streptococcus suis. PLoS ONE 4:e6072. 417 DOI: 10.1371 journal.pone.0006072.

418 Hui ACF, Ng KC, Tong PY, Mok V, Chow KM, Wu A, Wong LKS. 2005. Bacterial meningitis 419 in Hong Kong: 10-Years' experience. Clinical Neurology and Neurosurgery 107:366-370.

420 DOI: $10.1016 /$ j.clineuro.2004.10.006.

421 Huong VTL, Ha N, Huy NT, Horby P, Nghia HDT, Thiem VD, Zhu X, Hoa NT, Hien TT,

422 Zamora J, Schultsz C, Wertheim HFL, Hirayama K. 2014. Epidemiology, clinical

423 manifestations, and outcomes of streptococcus suis infection in humans. Emerging

424 Infectious Diseases 20:1105-1114. DOI: 10.3201/eid2007.131594.

425 Jacobs AA, van den Berg AJ, Loeffen PL. 1996. Protection of experimentally infected pigs by 426 suilysin, the thiol-activated haemolysin of Streptococcus suis. The Veterinary record $427 \quad 139: 225-8$. 
428 Jeong J, Kim G, Moon C, Kim HJ, Kim TH, Jang J. 2018. Pathogen Box screening for hit

429 identification against Mycobacterium abscessus. PLoS ONE 13. DOI:

$430 \quad$ 10.1371/journal.pone.0195595.

431 Lau H, Ferlan JT, Hertle Brophy V, Rosowsky A, Sibley CH. 2001. Efficacies of Lipophilic 432 Inhibitors of Dihydrofolate Reductase against Parasitic Protozoa. 45:187-195. DOI:

433 10.1128/AAC.45.1.187-195.2001.

434 Lun Z-R, Wang Q-P, Chen X-G, Li A-X, Zhu X-Q. 2007. Streptococcus suis: an emerging 435 zoonotic pathogen. The Lancet Infectious Diseases 7:201-209. DOI: 10.1016/S1473$436 \quad 3099(07) 70001-4$

437 Maneerat K, Yongkiettrakul S, Kramomtong I, Tongtawe P, Tapchaisri P, Luangsuk P, 438 Chaicumpa W, Gottschalk M, Srimanote P. 2013. Virulence Genes and Genetic Diversity of 439 Streptococcus suis Serotype 2 Isolates from Thailand. Transboundary and Emerging 440 Diseases 60:69-79. DOI: 10.1111/tbed.12157.

441 Marchler-Bauer A, Derbyshire MK, Gonzales NR, Lu S, Chitsaz F, Geer LY, Geer RC, He J, 442 Gwadz M, Hurwitz DI, Lanczycki CJ, Lu F, Marchler GH, Song JS, Thanki N, Wang Z, 443 Yamashita RA, Zhang D, Zheng C, Bryant SH. 2015. CDD: NCBI's conserved domain 444 database. Nucleic Acids Research 43:D222-D226. DOI: 10.1093/nar/gku1221.

445 McCarthy M. 2017. Woman dies after infection with bacteria resistant to all antibiotics available 446 in US. BMJ (Clinical research ed.) 356:j254. DOI: 10.1136/BMJ.J254.

447 McGann P, Snesrud E, Maybank R, Corey B, Ong AC, Clifford R, Hinkle M, Whitman T, Lesho 448 E, Schaecher KE. 2016. Escherichia coli Harboring mer-1 and bla CTX-M on a Novel IncF 449 Plasmid: First report of $m c r-1$ in the USA. Antimicrobial Agents and Chemotherapy 
451 Nelson RG, Rosowsky A. 2001. Dicyclic and tricyclic diaminopyrimidine derivatives as potent

452 inhibitors of Cryptosporidium parvum dihydrofolate reductase: Structure-activity and

453 structure-selectivity correlations. Antimicrobial Agents and Chemotherapy 45:3293-3303.

454 DOI: $10.1128 /$ AAC.45.12.3293-3303.2001.

455 O’Neill J. 2014. Review on Antimicrobial Resistance. Antimicrobial Resistance: Tackling a

456 Crisis for the Health and Wealth of Nations, 2014. 4. DOI: 10.1038/510015a.

457 Popov VM, Chan DCM, Fillingham YA, Atom Yee W, Wright DL, Anderson AC. 2006.

458 Analysis of complexes of inhibitors with Cryptosporidium hominis DHFR leads to a new

459 trimethoprim derivative. Bioorganic and Medicinal Chemistry Letters 16:4366-4370. DOI:

$460 \quad$ 10.1016/j.bmcl.2006.05.047.

461 Riley CB, Chidgey KL, Bridges JP, Gordon E, Lawrence KE. 2020. Isolates, antimicrobial

462 susceptibility profiles and multidrug resistance of bacteria cultured from pig submissions in

463 new zealand. Animals 10:1-16. DOI: 10.3390/ani10081427.

464 Rollo IM. 1955. The mode of action of sulphonamides, proguanil and pyrimethamine on

465 Plasmodium gallinaceum. J. Pharmacol 10. DOI: 10.1111/j.1476-5381.1955.tb00084.x.

466 Ruzauskas M, Vaskeviciute L. 2016. Detection of the mcr-1 gene in Escherichia coli prevalent in 467 the migratory bird species Larus argentatus. Journal of Antimicrobial Chemotherapy 468 71:2333-2334. DOI: 10.1093/jac/dkw245.

469 Sievers F, Higgins DG. 2014. Clustal Omega. In: Current Protocols in Bioinformatics. Hoboken, 470 NJ, USA: John Wiley \& Sons, Inc., 3.13.1-3.13.16. DOI: 10.1002/0471250953.bi0313s48. 
471 Songsungthong W, Yongkiettrakul S, Bohan LE, Nicholson ES, Prasopporn S, Chaiyen P,

472 Leartsakulpanich U. 2019. Diaminoquinazoline MMV675968 from Pathogen Box inhibits

473 Acinetobacter baumannii growth through targeting of dihydrofolate reductase. Scientific

474 Reports 9. DOI: 10.1038/s41598-019-52176-8.

475 Suankratay C, Intalapaporn P, Nunthapisud P, Arunyingmongkol K, Wilde H. 2004.

476 Streptococcus suis meningitis in Thailand. Southeast Asian Journal of Tropical Medicine 477 and Public Health 35:868-876.

478 Tan MF, Tan J, Zeng YB, Li HQ, Yang Q, Zhou R. 2020. Antimicrobial resistance phenotypes

479 and genotypes of Streptococcus suis isolated from clinically healthy pigs from 2017 to 2019

480 in Jiangxi Province, China. Journal of Applied Microbiology. DOI: 10.1111/jam.14831.

481 Tang J, Wang C, Feng Y, Yang W, Song H, Chen Z, Yu H, Pan X, Zhou X, Wang H, Wu B,

482 Wang H, Zhao H, Lin Y, Yue J, Wu Z, He X, Gao F, Khan AH, Wang J, Zhao G-P, Wang

483 Y, Wang X, Chen Z, Gao GF. 2006. Streptococcal Toxic Shock Syndrome Caused by

484 Streptococcus suis Serotype 2. PLoS Medicine 3:e151. DOI:

$485 \quad$ 10.1371/journal.pmed.0030151.

486 The Clinical and Laboratory Standards Institute. 2018. Methods for Dilution Antimicrobial 487 Susceptibility Tests for Bacteria That Grow Aerobically.

488 Thi Hoang Mai N, Thi Hoa N, Vu Thieu Nga T, Dieu Linh L, Thi Hong Chau T, Xuan Sinh D, 489 Hoan Phu N, Van Chuong L, Song Diep T, Campbell J, Dang Trung Nghia H, Ngoc Minh 490 T, Van Vinh Chau N, de Jong MD, Tran Chinh N, Tinh Hien T, Farrar J, Schultsz C. 2008. 491 Streptococcus suis Meningitis in Adults in Vietnam. Clinical Infectious Diseases 46:659492 667. DOI: $10.1086 / 527385$. 
493 Tirakarn S, Riangrungroj P, Kongsaeree P, Imwong M, Yuthavong Y, Leartsakulpanich U. 2012.

494 Cloning and heterologous expression of Plasmodium ovale dihydrofolate reductase-

495 thymidylate synthase gene. Parasitology International 61:324-332. DOI:

$496 \quad$ 10.1016/j.parint.2011.12.004.

497 Tonelli M, Naesens L, Gazzarrini S, Santucci M, Cichero E, Tasso B, Moroni A, Costi MP, 498 Loddo R. 2017. Host dihydrofolate reductase (DHFR)-directed cycloguanil analogues 499 endowed with activity against influenza virus and respiratory syncytial virus. European $500 \quad J o u r n a l$ of Medicinal Chemistry 135:467-478. DOI: 10.1016/j.ejmech.2017.04.070.

501 Varela NP, Gadbois P, Thibault C, Gottschalk M, Dick P, Wilson J. 2013. Antimicrobial 502 resistance and prudent drug use for Streptococcus suis. Animal health research reviews $503 \quad 14: 68-77$. DOI: $10.1017 /$ S1466252313000029.

504 Wertheim HFL, Nghia HDT, Taylor W, Schultsz C. 2009a. Streptococcus suis: An Emerging 505 Human Pathogen. Clinical Infectious Diseases 48:617-625. DOI: 10.1086/596763.

506 Wertheim HFL, Nghia HDT, Taylor W, Schultsz C, Taylor W, Schultsz C. 2009b. Streptococcus 507 suis: An Emerging Human Pathogen. Clinical Infectious Diseases 48:617-625. DOI:

$508 \quad 10.1086 / 596763$.

509 Wertheim HFL, Nguyen HN, Taylor W, Lien TTM, Ngo HT, Nguyen TQ, Nguyen BNT,

510 Nguyen HH, Nguyen HM, Nguyen CT, Dao TT, Nguyen TV, Fox A, Farrar J, Schultsz C,

511 Nguyen HD, Nguyen K Van, Horby P. 2009c. Streptococcus suis, an important cause of

512 adult bacterial meningitis in Northern Vietnam. PLoS ONE 4. DOI:

$513 \quad$ 10.1371/journal.pone.0005973.

514 Yongkiettrakul S, Maneerat K, Arechanajan B, Malila Y, Srimanote P, Gottschalk M, 
515 Visessanguan W. 2019. Antimicrobial susceptibility of Streptococcus suis isolated from

516 diseased pigs, asymptomatic pigs, and human patients in Thailand. BMC veterinary

517 research 15:5. DOI: 10.1186/s12917-018-1732-5.

518 Zhang Q, Nguyen T, McMichael M, Velu SE, Zou J, Zhou X, Wu H. 2015. New small-molecule 519 inhibitors of dihydrofolate reductase inhibit Streptococcus mutans. International Journal of

520 Antimicrobial Agents 46:174-182. DOI: 10.1016/j.ijantimicag.2015.03.015.

521 


\section{Figure 1}

MMV675968 inhibits Streptococcus suis dihydrofolate reductase (SSDHFR).

A) SsDHFR functions in E. coli PA414. Overnight cultures of E. coli PA414 carrying empty plasmids or expressing ECTS and SSDHFR were serially diluted and spotted onto LB plates supplemented with either $50 \mu \mathrm{g} / \mathrm{mL}$ thymidine or $0.2 \%(\mathrm{w} / \mathrm{v})$ arabinose. Overnight growth was recorded. A representative picture from three independent experiment is shown. B) MMV675968 inhibits SsDHFR in E. coli surrogate. E. coli PA414 expressing EcTS and SsDHFR

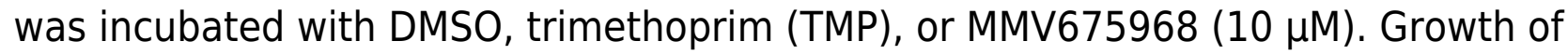
compound-treated $E$. coli surrogate was calculated as percent of untreated (100\%). C) Growth inhibition of E. coli surrogate expressing SsDHFR by MMV675968 can be rescued by thymidine supplement. E. coli PA414 expressing EcTS and SSDHFR was incubated with or without $10 \mu \mathrm{M}$ MMV675968 and with or without $50 \mu \mathrm{g} / \mathrm{mL}$ thymidine. Growth was monitored and compared with that of untreated control (100\%). D) MMV675968 inhibits SsDHFR activity. In vitro SsDHFR activity from clarified lysate of $E$. coli BL21(DE3) overexpressing SsDHFR was compared with that of E. coli BL21(DE3) harboring empty plasmid or in the presence of $1 \mu$ M MMV675968. E) S. suis growth inhibition by MMV675968 can be rescued by thymidine supplement. S. suis P1/7 and S. suis HE06 was incubated with or without $1 \mu \mathrm{M}$ MMV675968 and with or without $10 \mu \mathrm{g} / \mathrm{mL}$ thymidine. Growth was monitored and compared with that of untreated control $(100 \%)$. The graphs $(B-E)$ show mean \pm standard error of the mean (SEM) from three independent experiments. One-way ANOVA with Tukey's post test was performed to determine statistical significance compared with untreated control. *, P < $0.05 ; * *, \mathrm{P}<0.01 ; * * *, \mathrm{P}<0.0001 ; \mathrm{ns}$, not statistically significant. 
A.

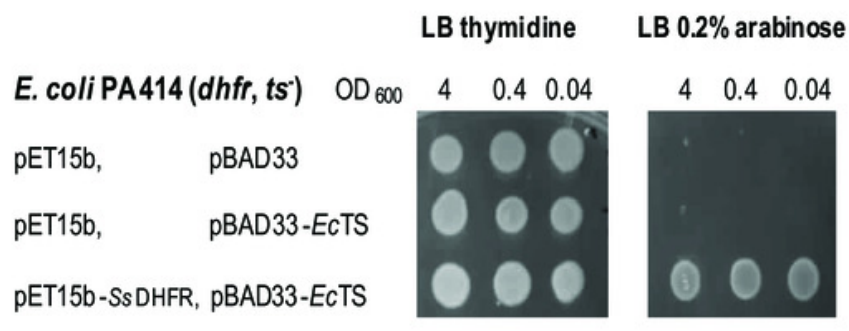

C.

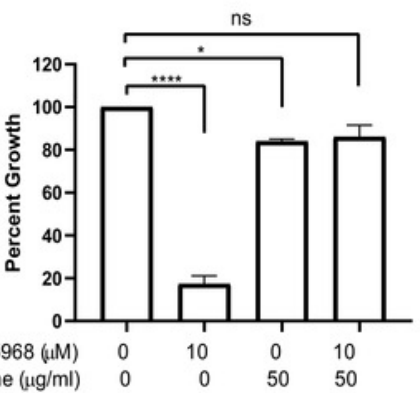

D.

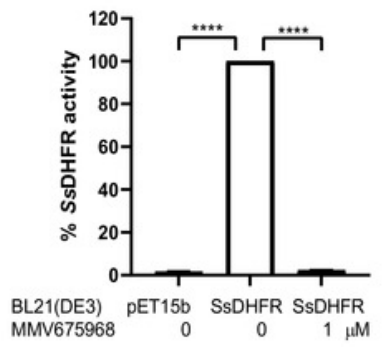

B.

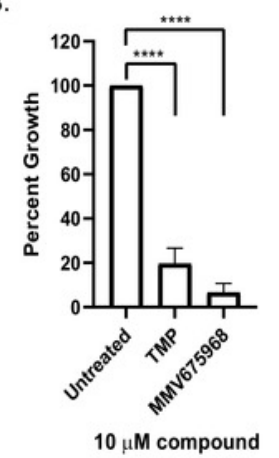

E.

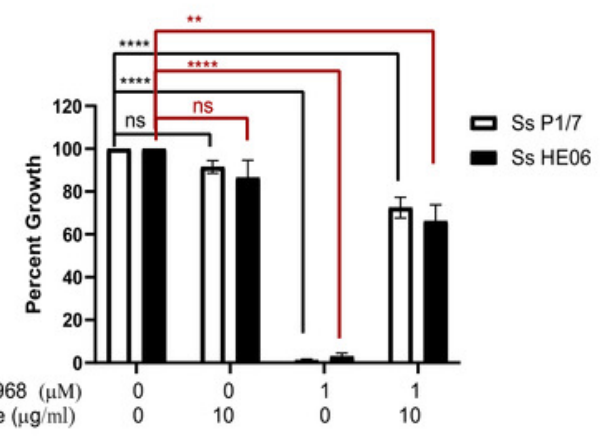




\section{Figure 2}

Efficacy of various dihydrofolate reductase (DHFR) inhibitors of Streptococcus suis DHFR (SSDHFR) and S. suis growth.

Structures of eight DHFR inhibitors tested in this study and grouped according to their inhibitory activity: A) ineffective inhibitors, B) moderately effective inhibitors, and C) highly effective inhibitors. D) SSDHFR activity in the presence of $1 \mu M$ DHFR inhibitors. E) Percent growth of S. suis P1/7 (white bar) and S. suis HE06 (black bar) after 18 hour incubation with $10 \mu \mathrm{M}$ of DHFR inhibitors. F) Percent growth of S. suis P1/7 (white bar) and S. suis HE06 (black bar) after 18 hour incubation with $1 \mu$ M inhibitors. PMX, pemetrexed; TMP, trimethoprim; PYR, pyrimethamine; MTX, methotrexate. The graphs show mean \pm standard error of the mean (SEM) from at least three independent experiments. One-way ANOVA with Tukey's post test was performed to determine statistical significance compared with untreated control. *, P $<0.05 ; * *, \mathrm{P}<0.01 ; * * *, \mathrm{P}<0.001 ; * * *, \mathrm{P}<0.0001$; ns, not statistically significant. For Fig. 2E-F, black asterisks show statistical significance between untreated and compound-treated S. suis P1/7 whereas red asterisks show statistical significance between untreated and compound-treated S. suis HE06. 
A.

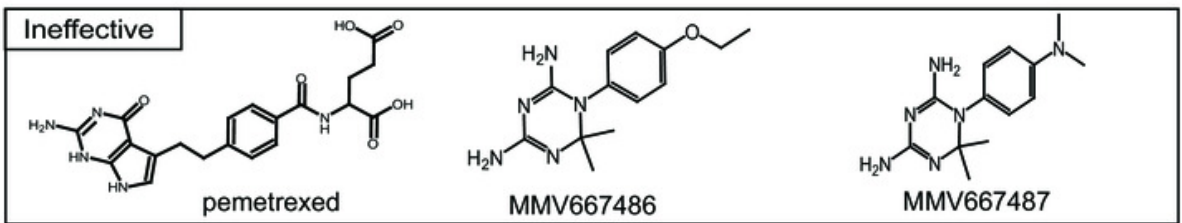

B.

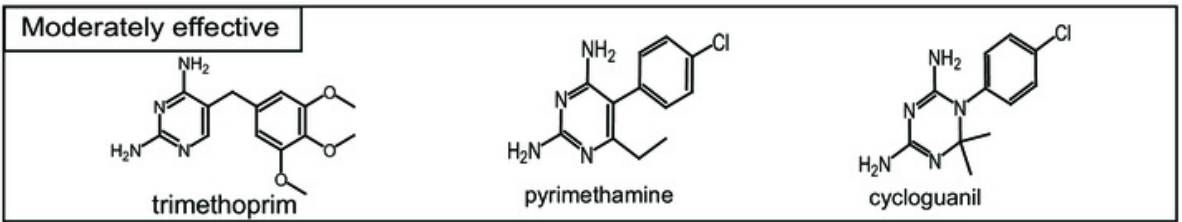

c.

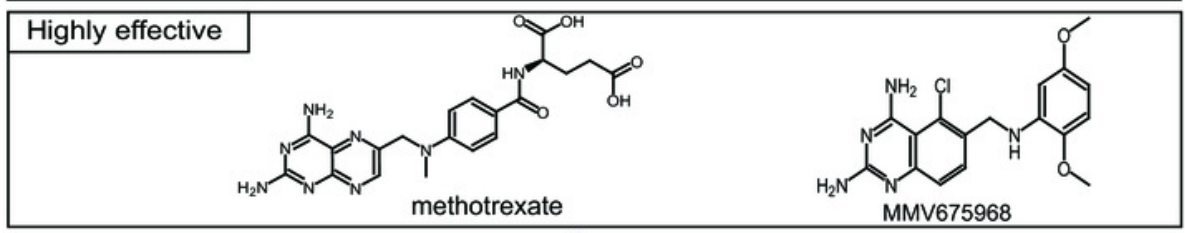

D.

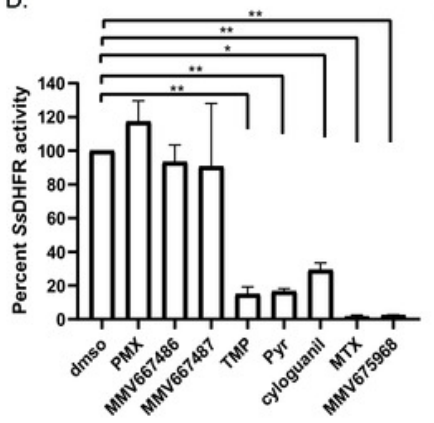

$1 \mu \mathrm{M}$ compound
E.

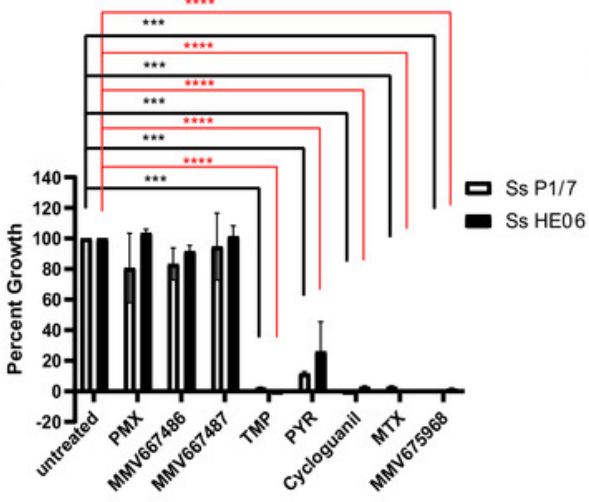

$10 \mu \mathrm{M}$ compound

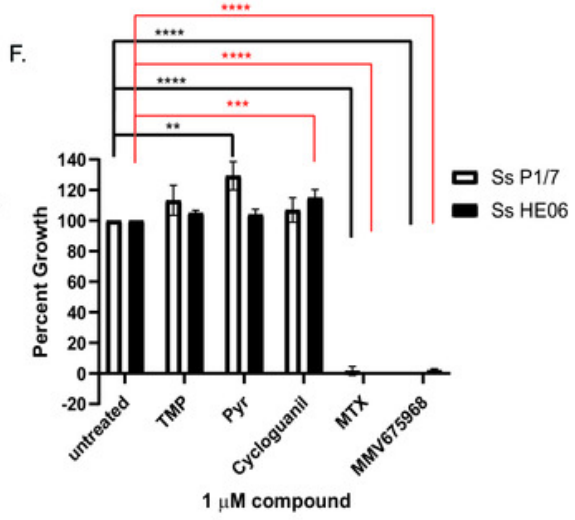

